\title{
Image-Guided Personalized Predictive Dosimetry by Artery-Specific SPECT/CT Partition Modeling for Safe and Effective ${ }^{90}$ Y Radioembolization
}

\author{
Yung Hsiang Kao ${ }^{1}$, Andrew Eik Hock Tan ${ }^{1}$, Mark Christiaan Burgmans ${ }^{2}$, Farah Gillian Irani ${ }^{2}$, Li Ser Khoo ${ }^{2}$, \\ Richard Hoau Gong $\mathrm{Lo}^{2}$, Kiang Hiong $\mathrm{Tay}^{2}$, Bien Soo $\mathrm{Tan}^{2}$, Pierce Kah Hoe Chow ${ }^{3,4}$, David Chee Eng Ng1, \\ and Anthony Soon Whatt Goh ${ }^{1}$ \\ ${ }^{1}$ Department of Nuclear Medicine and PET, Singapore General Hospital, Singapore; ${ }^{2}$ Department of Diagnostic Radiology, \\ Singapore General Hospital, Singapore; ${ }^{3}$ Department of General Surgery, Singapore General Hospital, Singapore; \\ and ${ }^{4}$ Office of Clinical Sciences, Duke-NUS Graduate Medical School, Singapore
}

Compliance with radiobiologic principles of radionuclide internal dosimetry is fundamental to the success of ${ }^{90} \mathrm{Y}$ radioembolization. The artery-specific SPECT/CT partition model is an image-guided personalized predictive dosimetric technique developed by our institution, integrating catheter-directed CT hepatic angiography (CTHA), 99mTc-macroaggregated albumin SPECT/CT, and partition modeling for unified dosimetry. Catheter-directed CTHA accurately delineates planning target volumes. SPECT/CT tomographically evaluates $99 \mathrm{~m} T \mathrm{Tc}-\mathrm{macro}-$ aggregated albumin hepatic biodistribution. The partition model is validated for ${ }^{90} \mathrm{Y}$ resin microspheres based on MIRD macrodosimetry. Methods: This was a retrospective analysis of ourearly clinical outcomes for inoperable hepatocellular carcinoma. Mapping hepatic angiography was performed according to standard technique with the addition of catheter-directed CTHA. ${ }^{99 m}$ Tc-MAA planar scintigraphy was used for liver-tolung shunt estimation, and SPECT/CT was used for liver dosimetry. Artery-specific SPECT/CT partition modeling was planned by experienced nuclear medicine physicians. Results: From January to May 2011, 20 arterial territories were treated in 10 hepatocellular carcinoma patients. Median follow-up was $21 \mathrm{wk}$ (95\% confidence interval [Cl], 12-50 wk). When analyzed strictly as brachytherapy, ${ }^{90} \mathrm{Y}$ radioembolization planned by predictive dosimetry achieved index tumor regression in 8 of 8 patients, with a median size decrease of $58 \%(95 \% \mathrm{Cl}, 40 \%-72 \%)$. Tumor thrombosis regressed or remained stable in 3 of 4 patients with baseline involvement. The best $\alpha$-fetoprotein reduction ranged from $32 \%$ to $95 \%$. Clinical success was achieved in 7 of 8 patients, including 2 by sublesional dosimetry, in 1 of whom there was radioembolization lobectomy intent. Median predicted mean radiation absorbed doses were 106 Gy $(95 \%$ $\mathrm{Cl}, 105-146 \mathrm{~Gy})$ to tumor, $27 \mathrm{~Gy}(95 \% \mathrm{Cl}, 22-33 \mathrm{~Gy})$ to nontumorous liver, and $2 \mathrm{~Gy}(95 \% \mathrm{Cl}, 1.3-7.3 \mathrm{~Gy})$ to lungs. Across all patients, tumor, nontumorous liver, and lungs received predicted $\geq 91$ Gy, $\leq 51$ Gy, and $\leq 16$ Gy, respectively, via at least 1 target arterial territory. No patients developed significant

\footnotetext{
Received Aug. 25, 2011; revision accepted Nov. 8, 2011.

For correspondence or reprints contact: Yung Hsiang Kao, Department of Nuclear Medicine and PET, Singapore General Hospital, Outram Rd., Singapore 169608.

E-mail: yung.h.kao@gmail.com

Published online Sep. 17, 2012.

COPYRIGHT @ 2012 by the Society of Nuclear Medicine, Inc.
}

toxicities within 3 mo after radioembolization. The median time to best imaging response was $76 \mathrm{~d}(95 \% \mathrm{Cl}, 55-114 \mathrm{~d})$. Median time to progression and overall survival were not reached. SPECT/CT-derived mean tumor-to-normal liver ratios varied widely across all planning target volumes (median, 5.4; 95\% $\mathrm{Cl}, 4.1-6.7)$, even within the same patient. Conclusion: Imageguided personalized predictive dosimetry by artery-specific SPECT/CT partition modeling achieves high clinical success rates for safe and effective ${ }^{90} \mathrm{Y}$ radioembolization.

Key Words: ${ }^{90} \mathrm{Y}$ radioembolization; ${ }^{90} \mathrm{Y}$ selective internal radiation therapy; catheter-directed CT hepatic angiography; $99 \mathrm{mTC}-$ macroaggregated albumin SPECT/CT; partition model MIRD macrodosimetry

J Nucl Med 2012; 53:559-566

DOI: 10.2967/jnumed.111.097469

A s a form of arterial territory-specific point-source brachytherapy, ${ }^{90} \mathrm{Y}$ radioembolization is always effective when delivered at the right location, in the right dose, and with the right intent. ${ }^{90} \mathrm{Y}$ radioembolization failure is invariably due to one or a combination of these 3 factors being incorrectly addressed. Responsibility for this triad of factors is shared among the interventional radiologist, the nuclear medicine physician, and the referring clinician. ${ }^{90} \mathrm{Y}$ radioembolization is complex, and a lack of coordinated care risks suboptimum outcomes.

Contemporary techniques, outcomes, and safety data of ${ }^{90} \mathrm{Y}$ radioembolization are well described in recent literature (1-8). Disregard for radiobiologic principles of arterial territory-specific ${ }^{90} \mathrm{Y}$ radionuclide internal dosimetry risks toxicity and fatality $(9,10)$. Since the $1980 \mathrm{~s}$, sectional imaging (e.g., CT) has revolutionized the planning and delivery of external-beam radiation therapy. However, to date, radiation planning for ${ }^{90} \mathrm{Y}$ radioembolization has yet to embrace modern imaging modalities such as catheterdirected CT hepatic angiography (CTHA) and SPECT with integrated low-dose CT (SPECT/CT). 
Catheter-directed CTHA delineates hepatic arterial territorial margins more accurately than digital subtraction angiography $(1,11)$. SPECT/CT is superior to both planar scintigraphy and SPECT for assessing intrahepatic biodistribution of ${ }^{99 \mathrm{~m}} \mathrm{Tc}-\mathrm{macroaggregated}$ albumin $\quad\left({ }^{99 \mathrm{~m}} \mathrm{Tc}-\right.$ MAA), and for estimating the tumor-to-normal liver (T/ $\mathrm{N})$ ratio (12-14). The partition model is a validated dosimetric method for ${ }^{90} \mathrm{Y}$ resin microspheres, scientifically superior to the body surface area (BSA) method $(5,10)$.

Accurate assessment of target liver volumes is critical because it directly affects radiation absorbed dose estimates. This is especially important when planning for selective (lobar) or superselective (segmental or subsegmental) ${ }^{90} \mathrm{Y}$ radioembolization. Catheter-directed CTHA refers to the acquisition of CT with direct intraarterial injection of dilute contrast medium through an angiographic catheter or microcatheter introduced into a hepatic lobar, segmental, or subsegmental artery via a percutaneous transfemoral arterial puncture $(1,11)$. This technique provides superior delineation of arterial territories compared with digital subtraction angiography, enabling accurate estimation of artery-specific perfused liver volumes (Supplemental Fig. 1; supplemental materials are available online only at http://jnm.snmjournals.org) $(1,11)$. Conventional ${ }^{90} \mathrm{Y}$ radioembolization is planned on the interventional radiologists' assessment of each patient's vascular anatomy (15). However, estimation of the perfused liver volume can be challenging. Hepatic vascular anatomy has many variations $(13,15,16)$. A main hepatic artery may supply several liver segments via its branches; conversely, a single segment may be supplied by more than a single arterial branch. Couinaud segments may be distorted by large tumors, previous liver surgery, or locoregional therapy-for example, ablation cavities. Aberrant arteries due to neoplastic recruitment from extrahepatic sources further complicate hepatic vascular anatomy. These factors make it difficult to reliably estimate the target volume of a diseased liver on the basis of digital subtraction angiography alone.

This problem may be overcome by catheter-directed CTHA. With the catheter tip placed in the target artery, CT performed at the time of intraarterial contrast injection provides superior delineation of the perfused liver volume, as compared with that shown under digital subtraction angiography. Each run of catheter-directed CTHA is specific for its catheter tip position. The use of catheterdirected CTHA shifts the planning emphasis away from a conventional lobe- or segment-based approach to a more patient-specific arterial territory-based approach. Catheterdirected CTHA accurately delineates each target arterial territory regardless of arterial anatomy, variant vasculature, or tissue distortion by tumor, surgery, or locally ablative treatments.

The role of SPECT/CT in diagnostic imaging and internal dosimetry is well established in nuclear medicine $(17,18)$. In the context of ${ }^{90} \mathrm{Y}$ radioembolization, ${ }^{99 \mathrm{~m}} \mathrm{Tc}-\mathrm{MAA}$ SPECT/ CT for preradioembolization assessment has a higher detec- tion rate for extrahepatic radiotracer activity and has greater impact on therapy planning than does planar scintigraphy $(14,19,20)$. Hepatic intraarterial ${ }^{99 m}$ Tc-MAA scintigraphy is a validated means of simulating the ${ }^{90} \mathrm{Y}$ radioembolization therapy field $(13,14)$. SPECT/CT-based dosimetry is superior to planar scintigraphy by tomographically resolving overlapping radiotracer activity, evaluating heterogeneous radiotracer uptake, and detecting activity in small lesions $(12,14,17)$. Phantom studies have shown that ${ }^{99 \mathrm{~m} T \mathrm{Tc}-\mathrm{MAA} \text { SPECT/CT }}$ volume measurements are accurate and reproducible (13). To date, no standardized technique exists for the calculation of SPECT/CT-based T/N ratios, although several methods have been described (21-23).

The partition model was developed and validated for ${ }^{90} \mathrm{Y}$ resin microspheres by Ho et al. in the 1990s $(24,25)$. It is one of several methods recommended by the manufacturer of ${ }^{90} \mathrm{Y}$ resin microspheres to calculate the desired ${ }^{90} \mathrm{Y}$ activity (26). Based on MIRD macrodosimetry, it partitions the lungs, tumor, and nontumorous liver into separate compartments for radiation dose modeling $(24,26)$. This model surpasses the commonly used BSA method by incorporating absolute tissue masses and patient-specific mean T/N ratios for personalized and scientifically sound estimates of mean radiation absorbed doses to each tissue compartment $(5,10,27)$. Technical differences between BSA methodology and partition modeling are discussed elsewhere, along with limitations and clinical implications (10).

The artery-specific SPECT/CT partition model is a unified technique of personalized predictive dosimetry developed by our institution for ${ }^{90} \mathrm{Y}$ radioembolization using resin microspheres. It integrates catheter-directed CTHA, ${ }^{99 \mathrm{~m} T c-M A A}$ SPECT/CT, and partition modeling for improved predictive radionuclide dosimetry (supplemental data). Its fundamental premise is to derive more accurate estimates of tissue masses and mean $\mathrm{T} / \mathrm{N}$ ratios to optimize partition modeling. The underlying principles and limitations of MIRD macrodosimetry remain unchanged. Through the use of artery-specific SPECT/CT partition modeling, predicted mean radiation absorbed doses specific to target arterial territories are independently calculated and physician-adjusted according to patient-specific circumstances (e.g., mean T/N ratio, liver-tolung shunt, liver reserve, prognosis, treatment intent, and potential treatment benefit). The overall process yields a personalized image-guided predictive radiation plan for safe and effective ${ }^{90} \mathrm{Y}$ radioembolization. This report provides a technical overview of this integrated dosimetric technique and details our early clinical outcomes in patients with inoperable hepatocellular carcinoma (HCC).

\section{MATERIALS AND METHODS}

The institutional review board waived the need to obtain informed consent for this retrospective analysis. The technique for using artery-specific SPECT/CT partition modeling to plan ${ }^{90} \mathrm{Y}$ radioembolization was implemented at our institution as a routine clinical service in January 2011. Five months after implementation, we had treated 22 patients with inoperable HCC. Of these, 10 were 
embargoed under an ongoing clinical trial. The treatment of 2 patients was planned by BSA methodology because of morphologically diffuse and infiltrative tumors that were below the resolution of ${ }^{99 \mathrm{~m}} \mathrm{Tc}-\mathrm{MAA}$ SPECT/CT, thus precluding reliable region-of-interest contouring. Both these patients were excluded (10). Treatment of the remaining 10 consecutive patients was planned by arteryspecific SPECT/CT partition modeling, and these patients were eligible for inclusion into this report. Retrospective review of hospital medical records was performed for these 10 patients until August 2011, the time at which the manuscript was prepared.

Patient and baseline disease characteristics were highly heterogeneous (Supplemental Tables 1 and 2). Median age was $59 \mathrm{y}$ (range, 48-65 y). There were 8 men and 2 women. Seven patients had chronic viral hepatitis. Five patients received prior therapy for $\mathrm{HCC}$, including 2 with previous ${ }^{90} \mathrm{Y}$ radioembolization planned by conventional planar partition modeling. Eight patients were ChildPugh A; the remainder were Child-Pugh B. Tumor extent varied widely across all patients and was mostly bilobar and multifocal. Eight patients had T4 disease according to the staging system of the United Network for Organ Sharing; none had nodal or distant metastases. Classification by the staging system of the Barcelona Clinic Liver Cancer was stage A for 1 patient, stage B for 3, stage C for 5, and stage $\mathrm{D}$ for 1 . Five patients had tumor vascular involvement.

\section{Technique Overview}

Mapping hepatic angiography and ${ }^{99 m}$ Tc-MAA injection were performed according to standard technique $(1,15)$. Prophylactic coil embolization of vessels at risk was performed either at mapping hepatic angiography or at ${ }^{90} \mathrm{Y}$ radioembolization, at the discretion of the interventional radiologist. The catheter tip position for ${ }^{99 m}$ Tc-MAA injection was decided by consensus between the interventional radiologist and nuclear medicine physician during mapping hepatic angiography. ${ }^{99 \mathrm{~m}} \mathrm{Tc}-\mathrm{MAA}$ was slowly hand-injected nonselectively (whole liver), selectively (lobar), or superselectively (segmental or subsegmental), depending on patient-specific circumstances (supplemental data). Patients were immediately transferred to the $\gamma$-camera suite for planar liver-to-lung shunt scintigraphy and SPECT/CT of the abdomen.

Technical aspects of artery-specific SPECT/CT partition modeling are detailed in the supplemental data. In essence, catheterdirected CTHA guides volume-of-interest delineation on ${ }^{99 \mathrm{~m}} \mathrm{Tc}-\mathrm{MAA}$ SPECT/CT to obtain arterial territory-specific tissue volumes and SPECT/CT-based mean T/N ratio estimates for improved partition modeling. The phrase planning target volume is introduced, adapted from external-beam radiation therapy. All treatments were planned by a team of experienced nuclear medicine physicians, and the final prescribed mean radiation absorbed doses were guided by published dose-response relationships (5). By partition modeling, the nuclear medicine physician had full control over predicted radiation absorbed dose estimates to tumor, nontumorous liver, and lungs within each planning target volume. The overall dosimetric aim was to balance the desired mean tumor radiation absorbed dose with collateral radiation injury, in accordance with the treatment intent. Illustrated online resources and worked examples are freely downloadable from our Web site to aid in understanding this dosimetric technique (supplemental data).

${ }^{90} \mathrm{Y}$ radioembolization was performed using resin microspheres (SIR-Spheres; Sirtex Medical Ltd.) within 2 wk of mapping hepatic angiography. Catheter tip placement was the same as that for the ${ }^{99 m}$ Tc-MAA injections. All patients received prophylactic omeprazole, $20 \mathrm{mg}$, twice daily before ${ }^{90} \mathrm{Y}$ radioembolization, and this treatment continued for at least $6 \mathrm{wk}$ after radioembolization. In accord with our institutional protocol, after ${ }^{90} \mathrm{Y}$ radioembolization all patients were observed overnight and discharged after bremsstrahlung planar scintigraphy of the lung and SPECT/ $\mathrm{CT}$ of the abdomen the following day.

\section{Terms and Definitions}

Postradioembolization bremsstrahlung SPECT/CT of the abdomen was used to determine technical success. Our bremsstrahlung scan protocol is detailed in the supplemental data. Technical success was achieved when all targeted tumors within planning target volumes showed satisfactory bremsstrahlung activity by visual assessment, in keeping with the radiation plan (28). The success of the technique was considered indeterminate when planning target volumes included subcentimeter tumors or tumors with ill-defined margins (e.g., infiltrative HCC), below bremsstrahlung SPECT/CT spatial resolution. A technical failure occurred when targeted tumors did not exhibit visually detectable focal bremsstrahlung activity, analogous to a geographic miss in the context of external-beam radiation therapy.

Clinical and biochemical toxicities within 3 mo after radioembolization were classified according to Common Terminology Criteria for Adverse Events (version 4.03) $(28,29)$. The nadir in serum $\alpha$-fetoprotein at any time after radioembolization indicated the best biochemical response.

To evaluate the effectiveness of ${ }^{90} \mathrm{Y}$ radioembolization strictly as a form of brachytherapy delivered at a single time point, the best imaging response was defined as the greatest change in size of the targeted index (largest) tumor between baseline and follow-up diagnostic sectional imaging. Clinical success was defined as any degree of regression of targeted tumors on follow-up diagnostic sectional imaging within planning target volumes, regardless of new tumors appearing within or outside planning target volumes (28).

Overall response according to the Response Evaluation Criteria in Solid Tumors (version 1.1), the criteria of the World Health Organization, and the 2-dimensional criteria of the European Association for the Study of the Liver were used to evaluate ${ }^{90} \mathrm{Y}$ radioembolization as a part of comprehensive multimodality therapy for inoperable HCC, without regard to planning target volumes $(28,30)$.

\section{Statistical Methods}

Data are presented as median and $95 \%$ confidence intervals (CI), where applicable. Using Bland-Altman analysis, the total desired ${ }^{90} \mathrm{Y}$ activities calculated by artery-specific SPECT/CT partition modeling were compared with those hypothetically derived by BSA methodology. The intraclass correlation coefficient was calculated, with values 0.8 or more considered excellent; $0.6-0.8$, good; 0.4-0.6, moderate; and less than 0.4 , poor. Statistical analysis was performed on Office Excel 2003 software (Microsoft Corp.) and SPSS software (version 17.0; SPSS Inc.).

\section{RESULTS}

The artery-specific SPECT/CT dosimetric plans of all 10 patients are summarized in Supplemental Table 10. There were a total of 20 radiation plans across 10 patients, that is, a single planning target volume per target arterial territory. Two patients underwent ${ }^{90} \mathrm{Y}$ radioembolization in a single arterial territory (Supplemental Fig. 2), 6 patients in 2 arterial territories, and 2 patients in 3 arterial territories (Fig. 1). 
Sublesional dosimetry was performed for 2 patients (Supplemental Figs. 3 and 4), in 1 of whom there was radioembolization lobectomy intent (Figs. 2 and 3). Four patients underwent prophylactic coil embolization of arteries at risk: 3 gastroduodenal, 2 accessory left gastric, 2 right gastric, 1 right inferior phrenic, 1 falciform, and 1 pancreaticoduodenal arcade. Technical success was achieved in 2 of 10 patients, indeterminate success in 7, and technical failure in 1 . Patient 8 was classified as a technical failure because of the absence of visually detectable focal bremsstrahlung activity in a targeted caudate tumor.

Median predicted mean radiation absorbed doses by arteryspecific SPECT/CT partition modeling were 106 Gy (95\% CI, 105-146 Gy) to tumor, $27 \mathrm{~Gy}$ (95\% CI, 22-33 Gy) to nontumorous liver, and 2 Gy $(95 \% \mathrm{CI}, 1.3-7.3 \mathrm{~Gy})$ to lungs. Across all patients, tumor, nontumorous liver, and lungs were predicted to have received $\geq 91 \mathrm{~Gy}, \leq 51 \mathrm{~Gy}$, and $\leq 16 \mathrm{~Gy}$, respectively, to at least 1 target arterial territory. The median artery-specific tumor and nontumorous liver masses were 156 g (95\% CI, 117-436 g) and 752 g (95\% CI, 513-833 g), respectively. The median liver-to-lung shunt estimated by planar 99mTc-MAA scintigraphy was 5.4\% (95\% CI, 4.3\%-9.0\%). The median ${ }^{90} \mathrm{Y}$ activity injected into an arterial territory was 0.6 GBq (95\% CI, 0.7-1.3 GBq). Patient 2 received a mean radiation absorbed dose of 93 Gy to the left lobe nontumorous liver for ${ }^{90} \mathrm{Y}$ radioembolization lobectomy (Supplemental Table 10; Fig. 2). There was good tumor response together with slow, progressive atrophy of the left lobe 6 mo after radioembolization, in keeping with the dosimetric intent. The right lobe volume, which received a mean radiation absorbed dose of 27 Gy to nontumorous liver, remained stable over time (Fig. 3).

SPECT/CT-based mean $\mathrm{T} / \mathrm{N}$ ratios varied widely across all planning target volumes (median 5.4; 95\% CI, 4.1-6.7) and even within the same patient (median intrapatient difference, $1.9 ; 95 \% \mathrm{CI}, 1.1-2.5)$. We could not find any predictive relationship between mean $\mathrm{T} / \mathrm{N}$ ratios and liver tissue masses. In a subanalysis, the total desired ${ }^{90} \mathrm{Y}$ activities calculated by artery-specific SPECT/CT partition modeling were compared with those hypothetically derived by BSA methodology. Bland-Altman analysis (Fig. 4) showed a wide $95 \%$ limit of agreement ranging from -1.12 to +1.41 , with only moderate correlation (intraclass correlation coefficient, $0.59 ; 95 \% \mathrm{CI},-0.71$ to 0.90 ).

${ }^{90} \mathrm{Y}$ radioembolization was well tolerated by all patients. None developed postradioembolization syndrome. All patients were ambulating freely by the next day and were discharged within $24 \mathrm{~h}$ after radioembolization. In 8 patients, serum
FIGURE 1. Example of artery-specific SPECT/CT partition modeling of 3 arterial territories. (A-C) Liver with multifocal HCC supplied by right $(A)$, middle $(B)$, and left $(C)$ hepatic arteries is depicted in digital subtraction angiography (top) and catheterdirected CTHA (bottom). (D) Regions of interest (ROI) are drawn on 99mTc-MAA SPECT/CT transaxial slices representing left (blue ROI), middle (orange $\mathrm{ROI}$ ), and right (green ROI) hepatic artery planning target volumes, implanted tumor (red ROI), and necrotic tumor (white ROI).
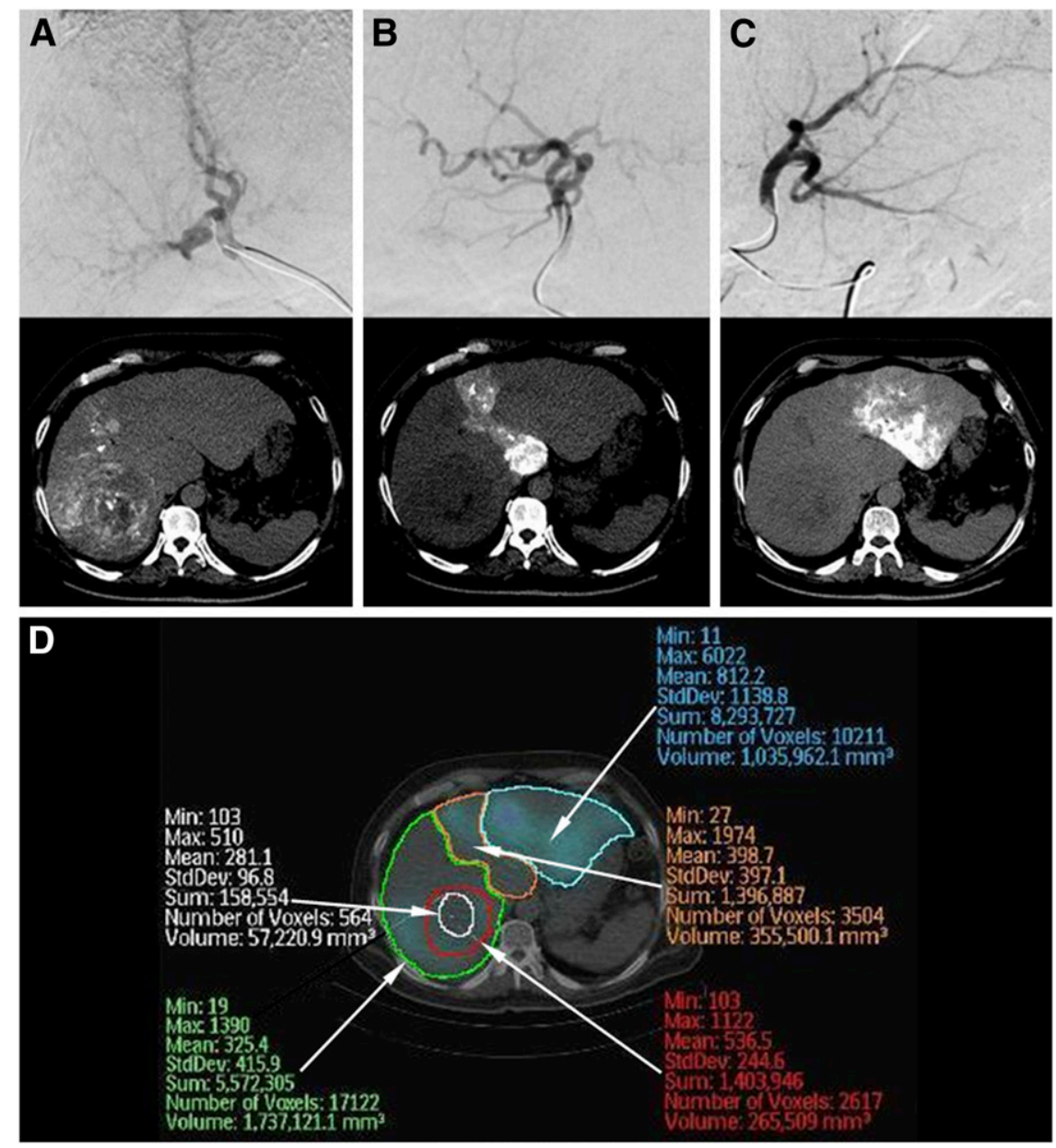

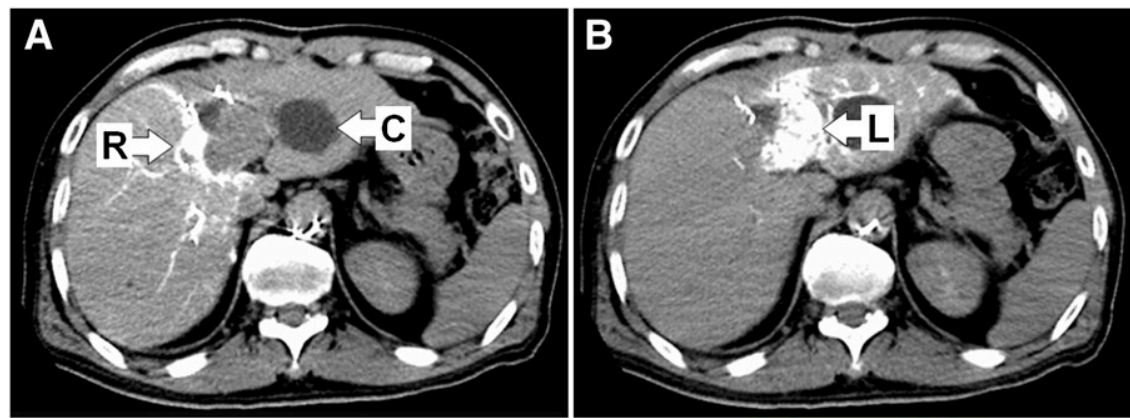

FIGURE 2. Example of sublesional dosimetry with left radioembolization lobectomy intent. Patient 2 had recurrent HCC at segment IV resection margin. Tumor was supplied by right and left hepatic arteries. (A and B) Catheter-directed CTHA depicts planning target volumes of right (A) and left (B) hepatic arteries, dividing dosimetric plan into 2 independent halves for sublesional dosimetry. Left lobe hypodensity is cyst. (C) ${ }^{99 m}$ Tc-MAA SPECT/CT shows good $\mathrm{T} / \mathrm{N}$ ratio (6.1) of lateral tumor
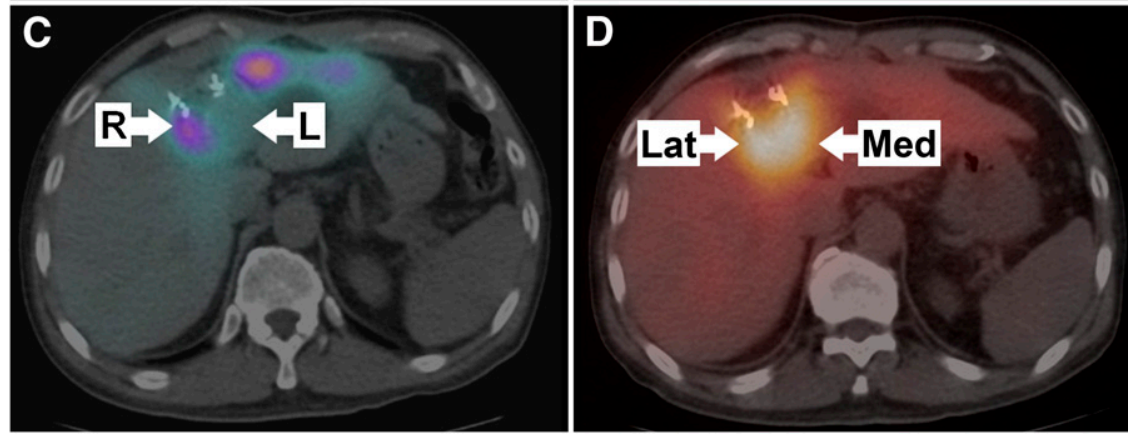
portion supplied by right hepatic artery but poor $\mathrm{T} / \mathrm{N}$ ratio (1.4) of medial tumor portion supplied by left hepatic artery. Dosimetric plan of left hepatic artery planning target volume was deliberately escalated beyond safe limits to achieve predicted mean radiation absorbed dose of 133 Gy to tumor and 93 Gy to nontumorous liver, where progressive atrophy of left lobe was the anticipated collateral effect, that is, left radioembolization lobectomy intent. (D) Postradioembolization bremsstrahlung SPECT/CT shows good tumoral activity in both lateral and medial tumor portions, indicating technical success. $\mathrm{C}=$ cyst; $\mathrm{L}=$ left hepatic artery; Lat $=$ lateral; Med $=$ medial; $\mathrm{R}=$ right hepatic artery

bilirubin, albumin, and alanine transaminase were measured within $24 \mathrm{~h}$ after radioembolization; none developed significant biochemical toxicities. The results are summarized in Supplemental Table 3.

At the time of this report, follow-up data were available for 8 patients. The median follow-up duration was $21 \mathrm{wk}$ (95\% CI, 12-50 wk). The other 2 patients were nonresidents, returned to their home country, and were lost to follow-up. Patient 3 was excluded from biochemical and survival analysis because of confounding medical issues (supplemental data) but was included in imaging analysis.

With the exception of patient 3 , all 7 of the other patients remained clinically well. None developed gastrointestinal complications or radiation pneumonitis. For 3 mo after radioembolization, there were no biochemical toxicities
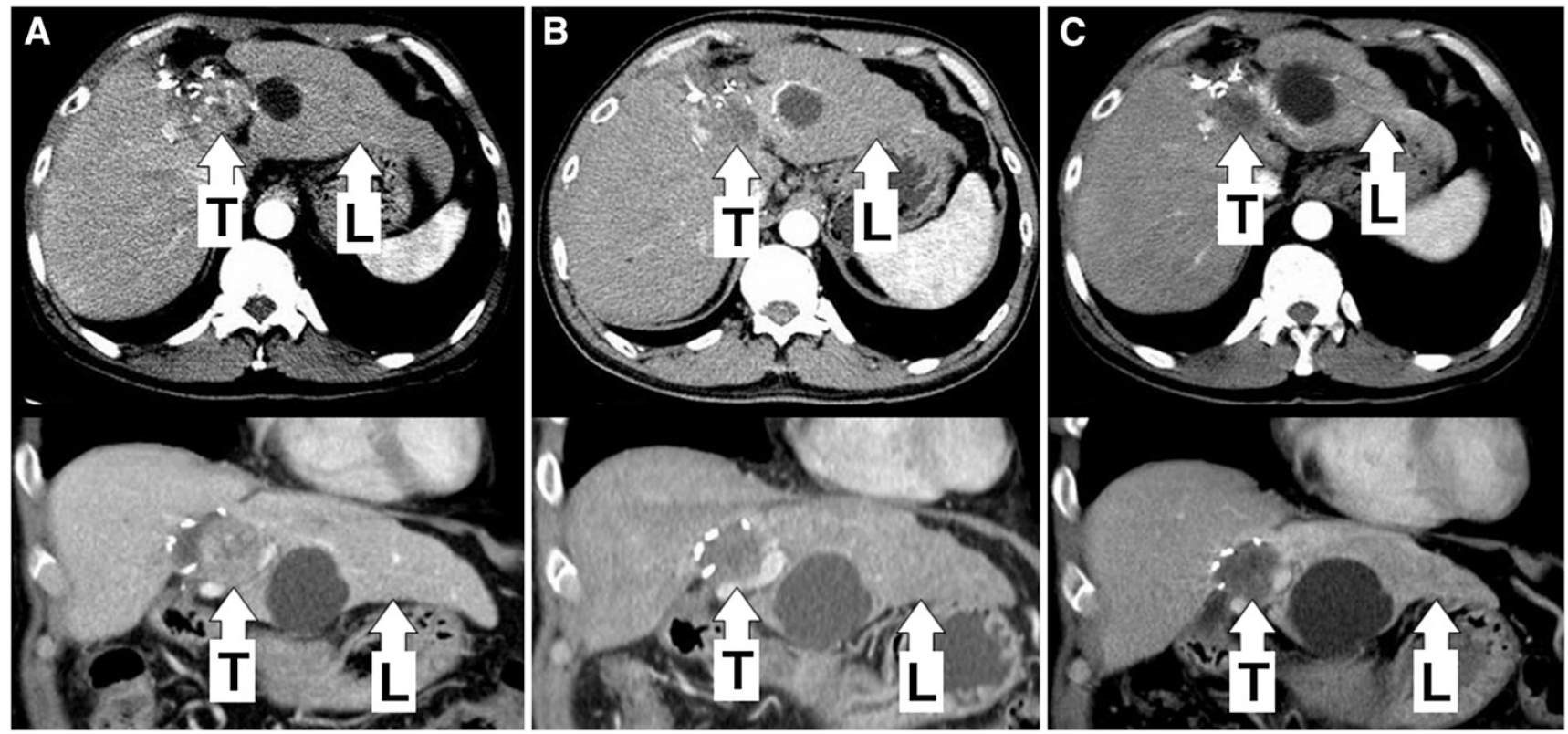

FIGURE 3. (A) Baseline triphasic CT scan of patient 2 shows recurrent $\mathrm{HCC}$ at segment IV resection margin. (B and C) Follow-up triphasic scans at $3(B)$ and $6(C)$ months after radioembolization show good tumor response (133 Gy), with progressive atrophy of left lobe (93 Gy), in keeping with left radioembolization lobectomy intent. Right lobe volume (27 Gy) remained stable. $\mathrm{L}=$ left lobe; $\mathrm{T}=$ tumor. 
FIGURE 4. Bland-Altman plot of ${ }^{90} \mathrm{Y}$ activities derived by artery-specific SPECT/ CT partition modeling vs. that hypothetically derived by BSA methodology.

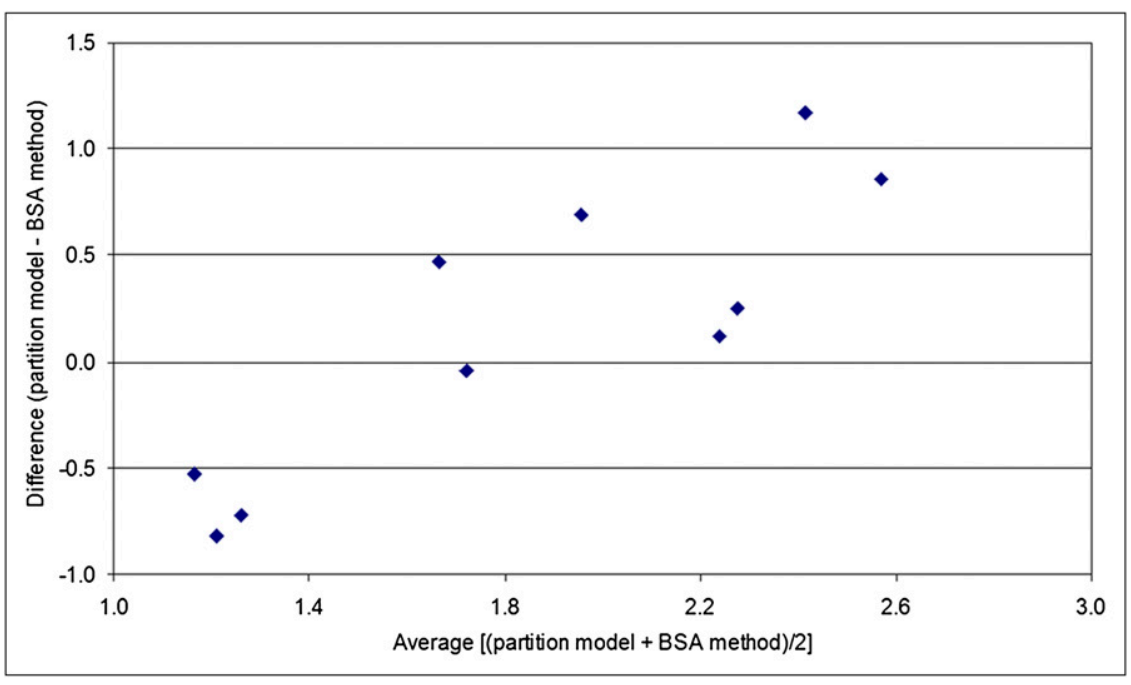

beyond grade 2 of the Common Terminology Criteria for Adverse Events (Supplemental Table 4). Postradioembolization serum $\alpha$-fetoprotein was available in 5 patients. There was an interval decrease in $\alpha$-fetoprotein in 3 patients, ranging from $32 \%$ to $95 \%$ (Supplemental Table 5). The remaining 2 patients had normal baseline $\alpha$-fetoprotein levels, which remained unelevated on follow-up.

The postradioembolization best imaging response was available for 8 patients (Supplemental Table 6). The median time to the best imaging response was $76 \mathrm{~d}(11 \mathrm{wk} ; 95 \% \mathrm{CI}$, 55-114 d). When ${ }^{90} \mathrm{Y}$ radioembolization was analyzed strictly as a brachytherapy, all 8 of 8 patients showed regression of the index tumor, with a median size decrease of $58 \%$ (95\% CI, 40\%-72\%). None developed new tumors within planning target volumes. Seven of 8 patients achieved clinical success at the time of best imaging response. Patient 1, who had infiltrative HCC, was classified as a clinical failure because of progression of existing portal vein tumor thrombosis despite significant regression of the index tumor (Supplemental Fig. 5). Otherwise, tumor thrombosis regressed or remained stable in 3 of 4 patients with baseline tumor vascular involvement.

At the time of best imaging response, extrahepatic metastases were discovered in 3 of 8 patients, involving the lungs and adrenal gland. When ${ }^{90} \mathrm{Y}$ radioembolization was analyzed as part of a comprehensive multimodality treatment plan, partial response was achieved in 3 of 8 , stable disease in 1 of 8 , and progressive disease in 4 of 8 patients (Supplemental Table 6) - consistent across all 3 classifications (Response Evaluation Criteria in Solid Tumors, World Health Organization criteria, and 2-dimensional criteria of the European Association for the Study of the Liver). Median imaging time to progression and median overall survival were not reached at the time of this report (Supplemental Table 7). Patient 1 died 13 wk after radioembolization and had an overall survival of $95 \mathrm{wk}$ ( $22 \mathrm{mo}$ ) under comprehensive multimodality care; all 7 of the other patients are still alive.

\section{DISCUSSION}

The aim of personalized predictive dosimetry is to guide decisions on radionuclide therapy in order to avoid the use of futile therapy and achieve the maximum tumor radiation absorbed dose while minimizing collateral radiation injury to normal tissue.

Precise predictive dosimetry has the potential to yield many benefits for cancer therapy, and the need for further research into internal dosimetry has recently been emphasized (31). To our knowledge, the artery-specific SPECT/ CT partition model is the first to integrate catheter-directed CTHA, ${ }^{99 m}$ Tc-MAA SPECT/CT, and partition modeling (MIRD) into a unified, accurate, and practical form of image-guided personalized predictive dosimetry for ${ }^{90} \mathrm{Y}$ radioembolization.

Our results show that a $100 \%$ tumor response rate could be achieved when the predicted mean tumor radiation absorbed dose was at least 91 Gy to a planning target volume. There were no significant toxicities, with predicted mean radiation absorbed doses to nontumorous liver and lungs of $\leq 51$ Gy and $\leq 16$ Gy, respectively. This finding highlights the power of predictive radionuclide dosimetry to achieve desired outcomes when planned in accordance with dose-response relationships. The capability of artery-specific SPECT/CT partition modeling is best exemplified by patient 2 , in whom sublesional dosimetry was successfully planned with ${ }^{90} \mathrm{Y}$ radioembolization lobectomy intent (Figs. 2 and 3).

${ }^{90} \mathrm{Y}$ radioembolization is a point-source, continuous lowdose-rate brachytherapy delivered at a single time point. Hence, its true therapeutic efficacy can be revealed only by analyzing outcomes in the context of planning target volumes and technical success. Therefore, the postradioembolization appearance of new tumors within a planning target volume should not be misconstrued as a clinical failure. Such lesions may represent new metastases, preexisting micrometastases that have enlarged, or de novo tumors arising from cirrhotic liver, all of which have little or no bearing on ${ }^{90} \mathrm{Y}$ radio- 
embolization as brachytherapy delivered at a single time point. It follows that when applying standard response evaluation criteria (e.g., Response Evaluation Criteria in Solid Tumors), which take into account new tumors and distant metastases, one should analyze ${ }^{90} \mathrm{Y}$ radioembolization brachytherapy as part of comprehensive multimodality care and not in isolation.

The application of ${ }^{90} \mathrm{Y}$ radioembolization without due regard for radiobiologic principles is akin to flying an aircraft without guidance from air traffic control. Since most patients with inoperable HCC have a limited prognosis, ${ }^{90} \mathrm{Y}$ radioembolization should be carefully planned using scientifically sound methods (e.g., MIRD) to achieve the maximum desired effect for optimal, personalized cancer therapy. Our dosimetric data showed wide inter- and intrapatient variations in SPECT/CT-based mean T/N ratios (Supplemental Table 10), emphasizing the importance of having a personalized dosimetric approach to ${ }^{90} \mathrm{Y}$ radioembolization. The assumption of a standard T/N ratio for the sake of dosimetric simplification may result in over- or undertreatment and confounds data analyses because reliable dose-response relationships cannot be established or verified (10). Despite its popularity, the BSA method has a questionable radiobiologic basis and is scientifically inferior to MIRD methodology $(5,10)$. One must be cognizant that the BSA method was first published by van Hazel et al. for whole-liver ${ }^{90} \mathrm{Y}$ radioembolization to previously untreated colorectal liver metastasis, not HCC (32). These patients did not have chronic liver disease, prior liver resection, local ablation, or selective or superselective ${ }^{90} \mathrm{Y}$ radioembolization. Furthermore, colorectal liver metastases are rarely bulky enough to distort liver anatomy. These features are often present in HCC, and therefore they cast doubt on the validity, safety, and efficacy of the use of BSA methodology for HCC. The fact that our data show a lack of agreement in total ${ }^{90} \mathrm{Y}$ activities derived by artery-specific SPECT/CT partition modeling versus the BSA method is further evidence against the routine use of BSA methodology (Fig. 4).

In this report, postradioembolization bremsstrahlung SPECT/CT was used to determine technical success. Success was indeterminate in 7 of 10 patients, all of whom had either subcentimeter tumors or tumors with ill-defined margins. This finding highlights the low spatial resolution of bremsstrahlung SPECT/CT as a technical limitation by indirectly imaging ${ }^{90} \mathrm{Y}$ biodistribution using scatter radiation. It may be possible for PET to overcome this limitation by coincidence imaging of ${ }^{90} \mathrm{Y}$ internal pair production (33). This possibility is currently under investigation at our institution.

${ }^{90} \mathrm{Y}$ microspheres, once implanted, remain permanently in place and decay to infinity in situ. This simplifies the dosimetric process because time-activity curves need not be obtained, unlike in systemic radionuclide therapy. Future ${ }^{90} \mathrm{Y}$ radioembolization dosimetric techniques must improve accuracy in several areas: delineation of arterial territory target volumes, microparticle simulation and biodistribution assessment, and predictive radiation dose-response model- ing. For example, future development of positron-labeled microspheres in place of ${ }^{99 \mathrm{~m}} \mathrm{Tc}-\mathrm{MAA}$ may increase the accuracy of liver-to-lung shunt calculation and of simulation of hepatic microsphere biodistribution and improve predictive radiation modeling by voxel- or Monte-Carlo-based techniques $(34,35) .{ }^{90} \mathrm{Y}$ radioembolization will also benefit from a wealth of experience if radiobiologic models based on external-beam radiation therapy (e.g., linear quadratic model and normal tissue complication probability model) and radiation-planning techniques (e.g., dose-volume histogram) can be meaningfully translated into radionuclide dosimetry models (e.g., MIRD) and vice versa. Application of the concept of biologically effective dose (BED) into ${ }^{90} \mathrm{Y}$ radioembolization dosimetry may achieve this aim (36).

\section{CONCLUSION}

Compliance with radiobiologic principles of radionuclide internal dosimetry is fundamental to ${ }^{90} \mathrm{Y}$ radioembolization success. Image-guided personalized predictive dosimetry by artery-specific SPECT/CT partition modeling achieves high clinical success rates for safe and effective ${ }^{90} \mathrm{Y}$ radioembolization.

\section{DISCLOSURE STATEMENT}

The costs of publication of this article were defrayed in part by the payment of page charges. Therefore, and solely to indicate this fact, this article is hereby marked "advertisement" in accordance with 18 USC section 1734.

\section{ACKNOWLEDGMENTS}

We thank Stephanie M.C. Fook, biostatistician, Division of Clinical Research, Singapore General Hospital, for assisting with the statistical analysis. No potential conflict of interest relevant to this article was reported.

\section{REFERENCES}

1. Salem R, Lewandowski RJ, Sato KT, et al. Technical aspects of radioembolization with ${ }^{90} \mathrm{Y}$ microspheres. Tech Vasc Interv Radiol. 2007;10:12-29.

2. Gaba RC, Lewandowski RJ, Kulik LM, et al. Radiation lobectomy: preliminary findings of hepatic volumetric response to lobar yttrium-90 radioembolization. Ann Surg Oncol. 2009;16:1587-1596.

3. Riaz A, Lewandowski RJ, Kulik LM, et al. Complications following radioembolization with yttrium-90 microspheres: a comprehensive literature review. $J$ Vasc Interv Radiol. 2009;20:1121-1130.

4. Ahmadzadehfar H, Biersack HJ, Ezziddin S. Radioembolization of liver tumors with yttrium-90 microspheres. Semin Nucl Med. 2010;40:105-121.

5. Lau WY, Kennedy AS, Kim YH, et al. Patient selection and activity planning guide for selective internal radiotherapy with yttrium-90 resin microspheres. Int J Radiat Oncol Biol Phys. 2012; 82:401-407.

6. Salem R, Lewandowski RJ, Mulcahy MF, et al. Radioembolization for hepatocellular carcinoma using yttrium-90 microspheres: a comprehensive report of long-term outcomes. Gastroenterology. 2010;138:52-64.

7. Wang SC, Bester L, Burnes JP, et al. Clinical care and technical recommendations for ${ }^{90}$ yttrium microsphere treatment of liver cancer. $J$ Med Imaging Radiat Oncol. 2010;54:178-187.

8. Riaz A, Gates VL, Atassi B, et al. Radiation segmentectomy: a novel approach to increase safety and efficacy of radioembolization. Int J Radiat Oncol Biol Phys. 2011;79:163-171. 
9. Jakobs TF, Hoffmann RT, Fischer T, et al. Radioembolization in patients with hepatic metastases from breast cancer. J Vasc Interv Radiol. 2008;19: 683-690.

10. Kao YH, Tan EH, Ng CE, Goh SW. Clinical implications of the body surface area method versus partition model dosimetry for yttrium- 90 radioembolization using resin microspheres: a technical review. Ann Nucl Med. 2011;25: $455-461$.

11. Rhee TK, Omary RA, Gates V, et al. The effect of catheter-directed CT angiography on yttrium-90 radioembolization treatment of hepatocellular carcinoma. J Vasc Interv Radiol. 2005;16:1085-1091

12. Pereira JM, Stabin MG, Lima FR, Guimarães MI, Forrester JW. Image quantification for radiation dose calculations: limitations and uncertainties. Health Phys. 2010;99:688-701.

13. Garin E, Rolland Y, Lenoir L, et al. Utility of quantitative Tc-MAA SPECT/CT for yttrium-labelled microsphere treatment planning: calculating vascularized hepatic volume and dosimetric approach. Int J Mol Imaging. July 28, 2011 [Epub ahead of print].

14. Kao YH, Tan EH, Teo TK, Ng CE, Goh SW. Imaging discordance between hepatic angiography versus Tc-99m-MAA SPECT/CT: a case series, technical discussion and clinical implications. Ann Nucl Med. 2011;25:669-676.

15. Salem R, Thurston KG. Radioembolization with ${ }^{90}$ yttrium microspheres: a stateof-the-art brachytherapy treatment for primary and secondary liver malignancies. Part 1: Technical and methodologic considerations. J Vasc Interv Radiol. 2006;17: 1251-1278.

16. Covey AM, Brody LA, Maluccio MA, Getrajdman GI, Brown KT. Variant hepatic arterial anatomy revisited: digital subtraction angiography performed in 600 patients. Radiology. 2002;224:542-547.

17. Flux G, Bardies M, Monsieurs M, Savolainen S, Strands SE, Lassmann M. The impact of PET and SPECT on dosimetry for targeted radionuclide therapy. Z Med Phys. 2006;16:47-59.

18. Patel CN, Chowdhury FU, Scarsbrook AF. Hybrid SPECT/CT: the end of "unclear" medicine. Postgrad Med J. 2009;85:606-613.

19. Hamami ME, Poeppel TD, Müller S, et al. SPECT/CT with ${ }^{99 \mathrm{~m}}$ Tc-MAA in radioembolization with ${ }^{90} \mathrm{Y}$ microspheres in patients with hepatocellular cancer. J Nucl Med. 2009;50:688-692.

20. Ahmadzadehfar H, Sabet A, Biermann K, et al. The significance of ${ }^{99 \mathrm{~m}} \mathrm{Tc}-\mathrm{MAA}$ SPECT/CT liver perfusion imaging in treatment planning for ${ }^{90} \mathrm{Y}$-microsphere selective internal radiation treatment. $J$ Nucl Med. 2010;51:1206-1212.

21. Gulec SA, Mesoloras G, Dezarn WA, McNeillie P, Kennedy AS. Safety and efficacy of Y-90 microsphere treatment in patients with primary and metastatic liver cancer: the tumor selectivity of the treatment as a function of tumor to liver flow ratio. J Transl Med. 2007;5:15-24.

22. Flamen P, Vanderlinden B, Delatte P, et al. Multimodality imaging can predict the metabolic response of unresectable colorectal liver metastases to radioembo- lization therapy with yttrium-90 labeled resin microspheres. Phys Med Biol. 2008;53:6591-6603.

23. Campbell JM, Wong CO, Muzik O, Marples B, Joiner M, Burmeister J. Early dose response to yttrium-90 microsphere treatment of metastatic liver cancer by a patient-specific method using single photon emission computed tomography and positron emission tomography. Int J Radiat Oncol Biol Phys. 2009; 74:313-320.

24. Ho S, Lau WY, Leung TW, et al. Partition model for estimating radiation doses from yttrium-90 microspheres in treating hepatic tumours. Eur J Nucl Med. 1996;23:947-952.

25. Ho S, Lau WY, Leung TW, Chan M, Johnson PJ, Li AK. Clinical evaluation of the partition model for estimating radiation doses from yttrium-90 microspheres in the treatment of hepatic cancer. Eur J Nucl Med. 1997;24:293-298.

26. Sirtex Medical Training Manual. New South Wales, Australia: Sirtex Medical Limited. Version TRN-US-03.

27. Gulec SA, Mesoloras G, Stabin M. Dosimetric techniques in ${ }^{90}$ Y-microsphere therapy of liver cancer: the MIRD equations for dose calculations. J Nucl Med. 2006;47:1209-1211.

28. Salem R, Lewandowski RJ, Gates VL, et al. Research reporting standards for radioembolization of hepatic malignancies. J Vasc Interv Radiol. 2011;22:265-278.

29. Common terminology criteria for adverse events (CTCAE): version 4.0. National Cancer Institute Web site. Available at: http://evs.nci.nih.gov/ftp1/CTCAE/ CTCAE_4.03_2010-06-14_QuickReference_8.5x11.pdf. Published May 28, 2009. Revised June 14, 2010. Accessed February 2, 2012.

30. Duke E, Deng J, Ibrahim SM. Agreement between competing imaging measures of response of hepatocellular carcinoma to yttrium-90 radioembolization. $J$ Vasc Interv Radiol. 2010;21:515-521.

31. Stabin MG, Sharkey RM, Siegel JA. RADAR commentary: evolution and current status of dosimetry in nuclear medicine. J Nucl Med. 2011;52:1156-1161.

32. Van Hazel G, Blackwell A, Anderson J, et al. Randomised phase 2 trial of SIRSpheres plus fluorouracil/leucovorin chemotherapy versus fluorouracil/leucovorin chemotherapy alone in advanced colorectal cancer. J Surg Oncol. 2004;88:78-85.

33. Gates VL, Esmail AA, Marshall K, Spies S, Salem R. Internal pair production of ${ }^{90} \mathrm{Y}$ permits hepatic localization of microspheres using routine PET: proof of concept. J Nucl Med. 2011;52:72-76.

34. Kennedy A, Dezarn W, Weiss A. Patient specific 3D image-based radiation dose estimates for ${ }^{90} \mathrm{Y}$ microsphere hepatic radioembolization in metastatic tumors. J Nucl Med Radiat Ther. 2011;2:1-8.

35. Gulec SA, Sztejnberg ML, Siegel JA. Hepatic structural dosimetry in ${ }^{90} \mathrm{Y}$ microsphere treatment: a Monte Carlo modeling approach based on lobular microanatomy. J Nucl Med. 2010;51:301-310.

36. Cremonesi M, Ferrari M, Bartolomei M. Radioembolisation with ${ }^{90}$ Y-microspheres: dosimetric and radiobiological investigation for multi-cycle treatment. Eur J Nucl Med Mol Imaging. 2008;35:2088-2096. 